\title{
An Improved Detection Scheme for Radar System
}

\author{
L. Donisha Greet and S. Hanis \\ P.G. Student (Communication Systems), Assistant Professor \\ Department of ECE \\ SSN College of Engineering
}

\begin{abstract}
A sequence of avian radar images containing the moving target is taken using an avian radar system. Radar image contains the complex background and the target (flying birds).

The main objective is to detect and track the moving target using Fast Independent Component Analysis (Fast ICA) and Hough Transform. Fast ICA is used to separate the target from the background of the radar image. Constant False Alarm Rate (CFAR) segmentation determines the threshold above which the return echoes have been originated from the moving target. Clutter Suppression is done by Conventional CFAR method. Low segmentation threshold is set to avoid missed targets. Finally, the position of bird is tracked by applying hough transform.
\end{abstract}

\section{Keywords}

Radar, target, Fast ICA, CFAR, threshold, clutter, track, Hough

\section{INTRODUCTION}

Visual monitoring techniques (e.g., bird censuses) provide information on the community of birds present on the airfield and how those communities change over a period of time. Visual monitoring provides only limited information on birdstrike threats. Also, the BASH (Bird-Animal Strike Hazard) managers are at a disadvantage because they can respond only after bird strikes occur [1]. So, avian radars are now used as a new tool to provide continuous sampling of bird activity that significantly supplements visual censuses [2]. Since bird strikes are still a threat to aviation safety, an improved detection scheme would help the BASH managers to act wisely so as to overcome the risks. With avian radar system, the target echo signal image is gathered and transmitted to the computer for further processing.

In this paper, a new detection scheme based on Fast Independent Component Analysis and Hough Transform have been used to detect the position of moving target.

\section{DETECTION AND TRACKING SCHEME}

Our avian radar system consists of a radar to collect the target echo signal. Hence, the image is gathered via image collecting card, and then transmitted to the computer for further processing. Through image processing of background subtraction, CFAR segmentation method and position determination by Hough transform, 2D position parameter data can be obtained. Target track information is then transferred to the BASH managers to act accordingly. Also, the data is stored in avian database for further analysis and research. The working block diagram of the system is shown in Figure 1

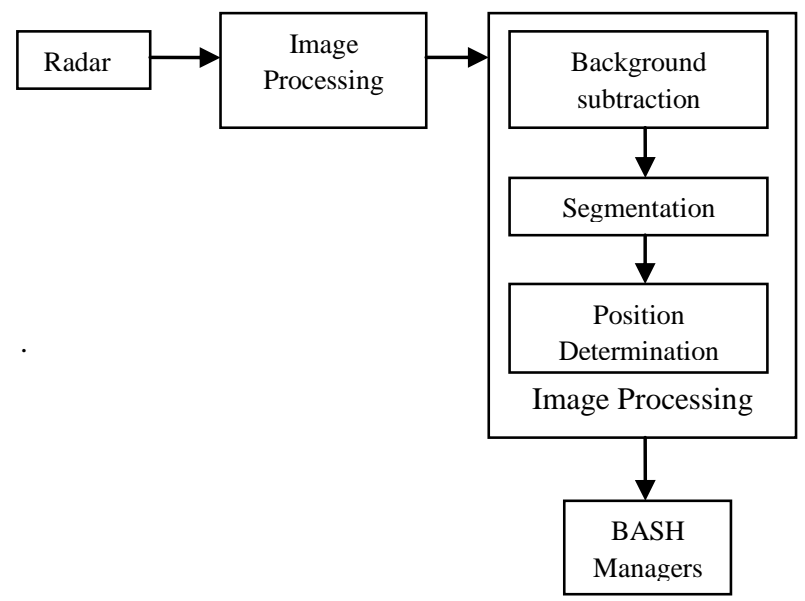

Figure 1. Avian Radar System

The avian radar image sequence containing the moving target is obtained after image collection. Image processing is done by background subtraction, segmentation and position determination. Fast Independent Component Analysis (Fast ICA) is applied to the image to separate the background and the moving target. Through background subtraction, the targets are detected. But there is a possibility that echoes being originated from different objects, called clutters. So, to reduce the unwanted clutter echoes, Conventional Constant False Alarm Rate detection is done [3]. The threshold is set so that the reflected echoes above this threshold are detected as target and those below this threshold belong to background. Clutter suppression is done by Conventional CFAR method. Hough transform for straight lines is applied to track the moving target. Image Processing is shown in Figure 2. 


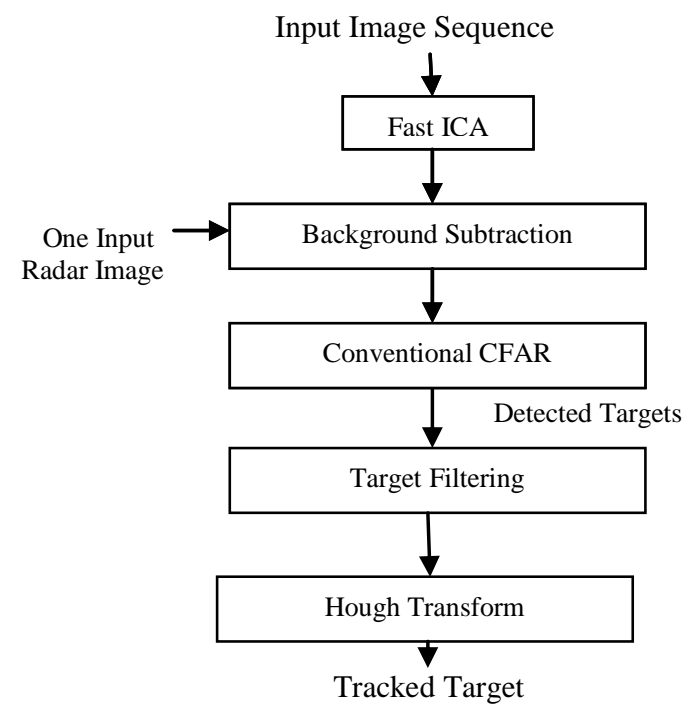

Figure 2. Detection and Tracking Scheme

\subsection{Fast Independent Component Analysis}

Fast Independent Component Analysis (Fast ICA) is a method for blind source separation. Blind Source Separation means separation of a set of signals from a set of mixed signals, without the aid of information or with little information about the source signals or the mixing process. Blind source separation relies on the assumption that the source signals may be statistically independent. The goal of Fast ICA is to find a linear representation of non-gaussian data so that the components are statistically independent. Before applying Fast ICA, some preprocessing has to be done [4].

\subsubsection{Centering and Whitening}

Centering is done by subtracting the input mixed signal by its mean so as to make the input mixed signal a zero mean variable. This preprocessing is made only to simplify the Fast ICA algorithm.

Whitening transforms the centered data to a new vector whose components are uncorrelated.

Let $\mathbf{x}$ be the input mixed signal and $\widetilde{\boldsymbol{x}}$ be the whitened signal. Whitening can be done by

$$
\tilde{\mathbf{x}}=\mathbf{E D}^{-1 / 2} \mathbf{E}^{T} \mathbf{x}
$$

where $\mathrm{E}$ is the orthogonal matrix of eigenvectors of $\mathbf{E}\left\{\mathbf{x} \mathbf{x}^{T}\right\}$ and $\mathrm{D}$ is the diagonal matrix of its eigen values, obtained after the Eigen Value Decomposition (EVD) of co-variance matrix $\mathbf{E}\left\{\mathbf{x x}^{T}\right\}=\mathbf{E D E}^{T}$.

The ICA model is shown in equation (2). Observed data denoted by $\mathbf{x}$ is a group of vectors with elements $x_{1} \ldots x_{n}$. Source data denoted by $\mathbf{s}$ cannot be observed directly. $\mathbf{M}$ is the mixing matrix.

$$
\mathbf{x}=\mathbf{M s}
$$

Let us assume that the components $\mathbf{s}$ are statistically independent. After estimating the matrix $\mathbf{M}$, its inverse say $\mathbf{W}$, can be computed and the independent components can be obtained by

$$
\mathbf{s}=\mathbf{W x}
$$

The Fast ICA is based on a fixed-point iteration scheme for finding a maximum of the non-gaussianity. It uses negentropy as a measure of non-gaussianity. Maximizing negentropy equals minimizing mutual information when estimating independence. Negentropy can be approximated by estimating the nongaussianity of the random variables directly [5].

The approximate function for negentropy measurement is

$$
\mathrm{N}_{\mathrm{g}}(\mathrm{Y})=\mathrm{E}[\mathrm{g}(\mathrm{y})]-\left\{\mathrm{E}\left[\mathrm{g}\left(\mathrm{Y}_{\text {gauss }}\right)\right]\right\}^{2}
$$

Where $g($.$) are some non-quadratic functions such as$

$$
\begin{aligned}
& \mathrm{g} 1(\mathrm{y})=\tanh (\mathrm{ay}) \\
& \mathrm{g} 2(\mathrm{y})=\mathrm{y} \exp \left(-\mathrm{ay}^{2} / 2\right) \\
& \mathrm{g} 3(\mathrm{y})=\mathrm{y}^{3}
\end{aligned}
$$

where $1<\mathrm{a}<2$, often taken as $\mathrm{a}=1$.

To estimate one of the independent components, consider a linear combination of the xi. Let us denote this by $y=w^{T} x$, where $\mathrm{w}$ is a vector to be determined. Initially, ' $\mathrm{w}$ ' is taken as a unit vector. Then, vector ' $w$ ' is updated by Newton's iteration for Fast ICA [6], [7].

The Fast ICA algorithm is as follows:

1. Choose an initial (e.g. random) weight vector $\mathbf{w}(\mathbf{0})$. Let $\mathbf{k}=\mathbf{1}$.

2. Let $\mathbf{w}(\mathbf{k})=\mathbf{E}\left\{\mathbf{x g}\left(\mathbf{w}^{\mathrm{T}}(\mathbf{k}-1) \mathbf{x}\right)\right\}-\mathbf{E}\left\{\mathbf{g} \square\left(\mathbf{w}^{\mathrm{T}}(\mathbf{k}-\mathbf{1}) \mathbf{x}\right)\right\} \mathbf{w}(\mathbf{k}-\mathbf{1})$

3. Let $\mathbf{w}=\mathbf{w}(\mathbf{k}) /\|\mathbf{w}(\mathbf{k})\|$

4. If not converged, go back to 2 .

Convergence means that the old and new values of $\mathrm{w}$ point in the same direction, i.e. their dot-product are equal to 1.

\subsection{Clutter Suppression by Conventional CFAR}

Clutter Suppression is done by Conventional CFAR method. Threshold is set to detect the moving target. In this paper, low threshold value of 3 is set to avoid missing of target but this may introduce more false alarms which have to be reduced. Due to the flexible body posture of birds, there may be errors because some target pixels are mistakenly included and some background pixels are mistakenly excluded. So to reduce this, a target filtering algorithm can be used [8], [9].

\subsection{Target Filtering}

Each and every type of target has to be defined to track them appropriately. For that, the number of pixels is taken into account to define each target. Initially, a single target is defined and is separated from all the other frames. Similarly, multiple targets can be found by defining each of them. Then, tracking algorithm is used to find the position change of the target. Hough transform is used for locating the target and calculating the distance as well.

\subsection{Tracking by Hough Transform}

The Hough transform is a technique used to find shapes in a binary digital image. By Hough Transform it is possible to find all kind of shapes that can be mathematical expressed, for instance lines, circles and ellipses, but only straight lines will be considered in our application [10]. If having a white pixel in a binary image, infinity many straight lines can go through that 
single pixel, and each of these lines can go through other white pixels in the same image, and the more white pixels on the same line the more is this line represented in the image. This is the principle of the Hough transform for straight lines.

If the distance $\rho$ (rho) is the distance from the origin to the line along a vector perpendicular to the line, and the angle $\theta$ (theta) is the angle between the $\mathrm{x}$-axis and the $\rho$ vector

$$
\rho=x \cos (\theta)+y \sin (\theta)
$$

The values that and $\rho$ can have are limited to: $\theta \varepsilon[0,180]$ degrees or $\theta \varepsilon[0, \pi]$ in radians, and $\rho \varepsilon[-D, D]$ where $D$ is the diagonal of the image.

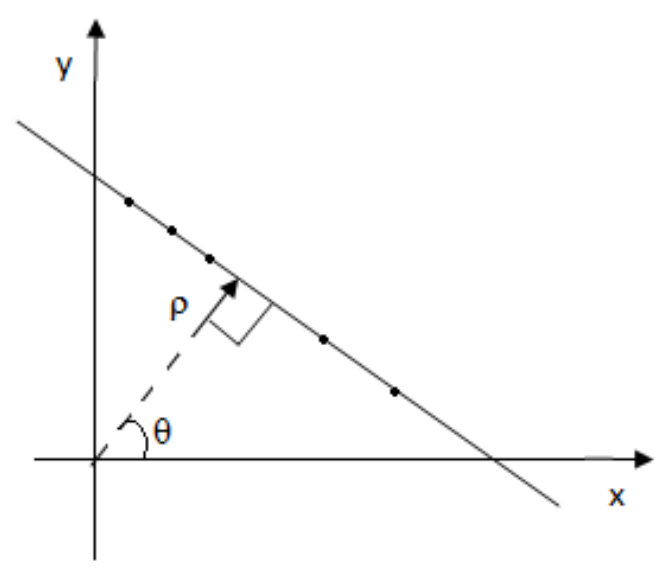

Figure 3. $(\rho, \theta)$ Line Parametrization

A line can then be transformed into a single point in the parameter space with the parameters $\theta$ and $\rho$, this is also called the Hough space.

If, instead of a line, having a pixel in an image with the position $(\mathrm{x}, \mathrm{y})$, infinity many lines can go through that single pixel. By using Equation 6, all these lines can be transformed into the Hough space, which gives a sinusoidal curve that is unique for that pixel. Doing the same for another pixel, gives another curve that intersect the first curve in one point, in the Hough space. This point represents the line, in the image space, that goes through both pixels. This can be repeated for all the pixels on the edges, in an edge detected image.

The result of the Hough transform is stored in a matrix called an accumulator. One dimension of this matrix is the theta values (angles) and the other dimension is the rho values (distances). Thus the distance and direction of travel of the bird are found.

Similarly, the distance of travel of the bird and direction of movement are found for all the birds. But, as the image shows the movement of a flock of birds, it is necessary to find the average distance and average velocity of their flight.

\subsection{Average Distance and Average Velocity}

Input image sequence is composed of multiple target i.e, a flock of birds. So this requires a filtration methodology to obtain their distance and direction of movement.
The simple logic that a flock of birds at higher altitude appear larger and those at a lower altitude appear smaller is taken into account. Four different targets are defined based on their levels of altitude. The targets at higher altitude are filtered first from each frame. Similarly, the targets at lower altitudes are also filtered and hough transform is applied to the filtered target to obtain the distance and direction of their movement. Finally, the average distance and average velocity are calculated.

\section{SIMULATION RESULTS}

A sequence of radar images are collected by using an avian radar system. Figure 4 shows the first image from the sequence of input radar images. Figure 5 shows the Fast ICA output for the input image. Figure 6 shows the detected targets after the background subtraction. Figure 7 shows the detected target using Convention CFAR. Figure 8 shows target1 from frame1. Figure 9 shows target 1 from frame2. Figure 10 shows the path of the moving target. Figure 11 shows the position of detected target.

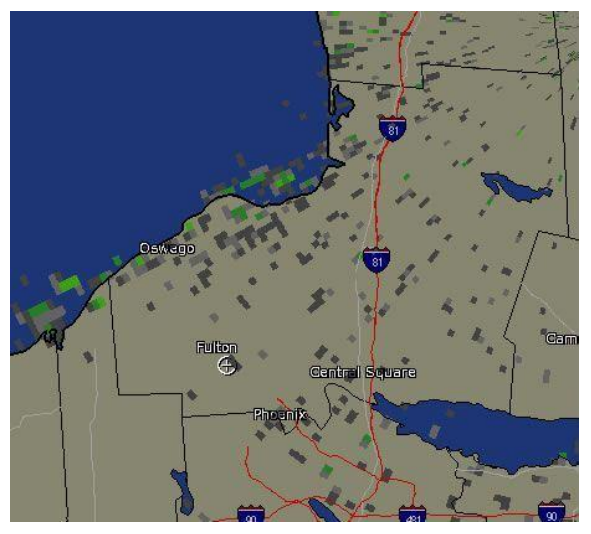

Figure 4. Input image

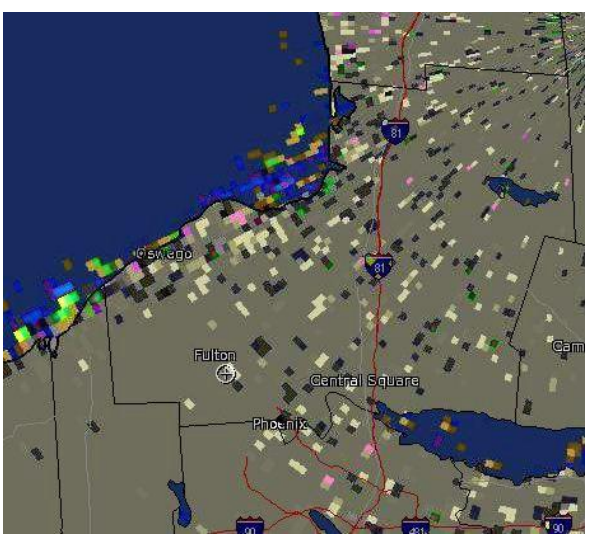

Figure 5. Fast ICA output 


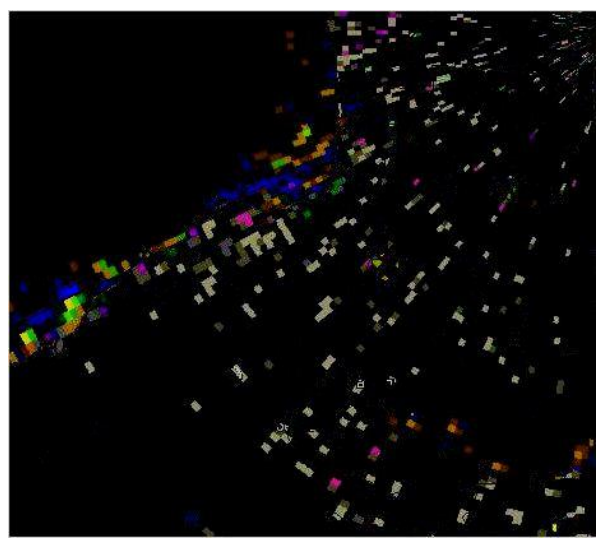

Figure 6. After Background Subtraction

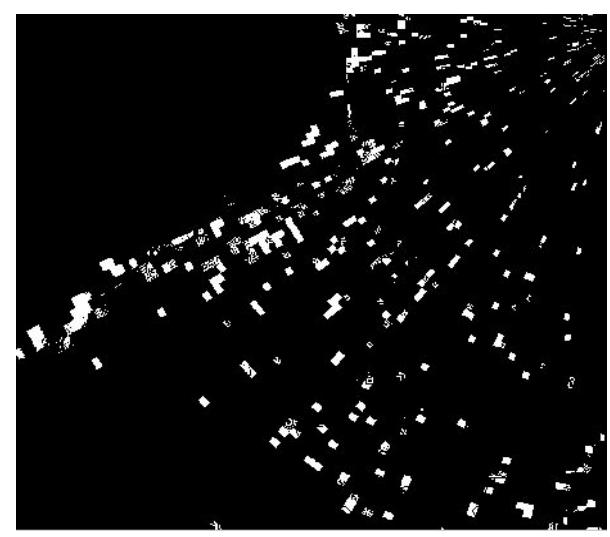

Figure 7. After clutter suppression using Conventional CFAR

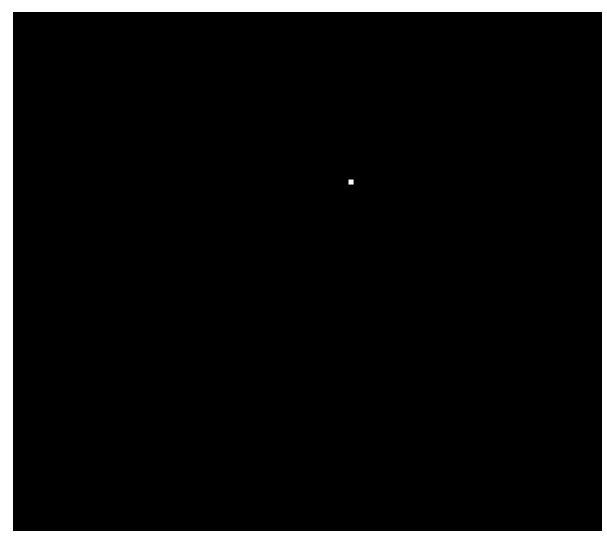

Figure 8. Extracted target1 from frame1

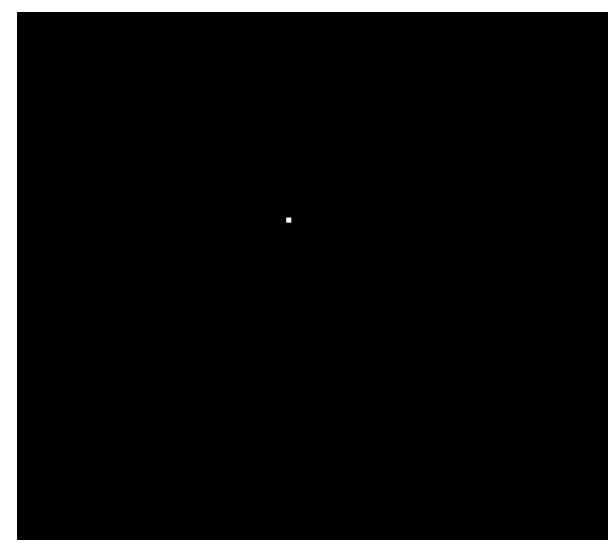

Figure 9. Extracted target1 from frame2

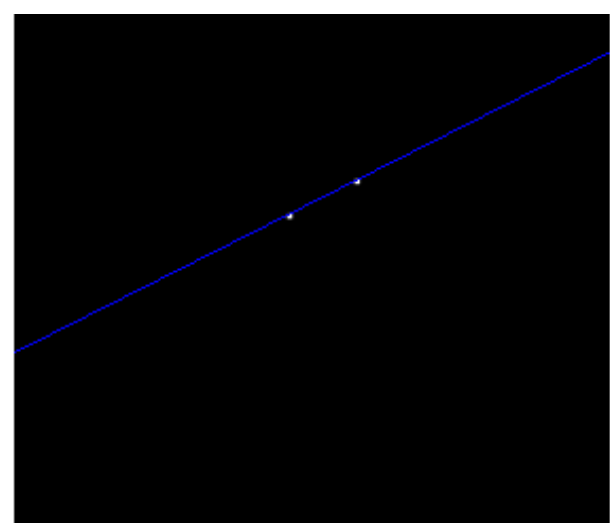

Figure 10. Path of the moving target

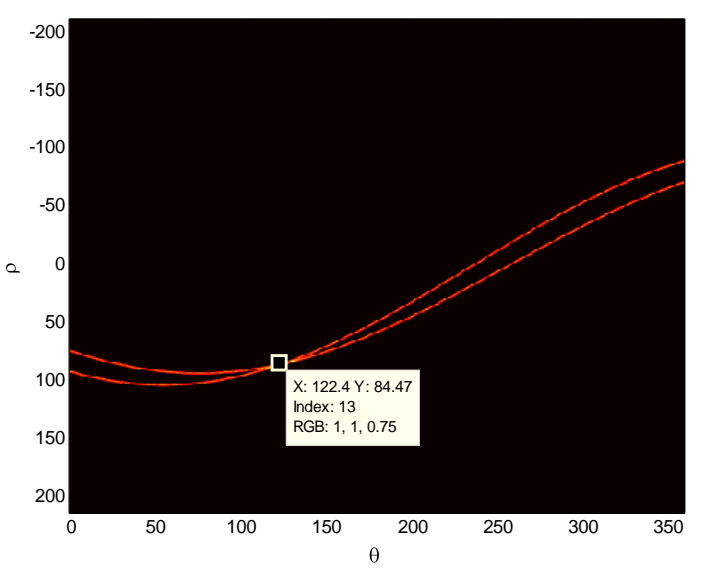

Figure 11. Position of Detected Target 
Table 1 gives the position of target 1 in $(\rho, \theta)$ for the first 5 frames.

\section{Table 1. POSITION OF TARGET 1}

\begin{tabular}{|c|c|c|}
\hline Frame No & $\mathbf{P}(\mathbf{m})$ & $\boldsymbol{\Theta}(\mathbf{d e g})$ \\
\hline 1 & 120.6 & 91.76 \\
\hline 2 & 84.47 & 122.4 \\
\hline 3 & 77.2 & 36.86 \\
\hline 4 & 71.12 & 60.39 \\
\hline 5 & 19.74 & 282.4 \\
\hline
\end{tabular}

Each frame has a time difference of 6 minutes.

Table 2 gives the average distance and average velocity of the birds.

Table 2. AVERAGE DISTANCE AND AVERAGE VELOCITY

\begin{tabular}{|c|c|c|}
\hline \multicolumn{3}{|c|}{ Birds at highest altitude } \\
\hline Frame No & $\begin{array}{c}\text { Average } \\
\text { Distance(m) }\end{array}$ & $\begin{array}{c}\text { Average } \\
\text { Velocity(kmph) }\end{array}$ \\
\hline $1-2$ & 28.2131 & 10.1567 \\
\hline $2-3$ & 30.0639 & 10.8230 \\
\hline $3-4$ & 38.6420 & 13.9111 \\
\hline $4-5$ & 39.1310 & 14.0871 \\
\hline
\end{tabular}

Similarly, the average distance and average velocity are found for birds at higher altitude, lower altitude and lowest altitude.

\section{CONCLUSION}

A new detection and tracking scheme is thus developed for avian radar system. The target and background are separated using Fast Independent Component Analysis (Fast ICA). Clutter suppression is done by Constant False Alarm Rate (CFAR) segmentation. Initially, a single bird target is extracted and tracked by using Hough Transform and the position of the bird is found for a short period of time. Results obtained by processing radar images containing birds have been shown. The average distance and average velocity are calculated for birds at highest altitude, higher altitude, lower altitude and lowest altitude.

\section{ACKNOWLEDGEMENT}

The authors wish to thank the management of SSN college of Engineering, Chennai for providing all the computational facilities to carry out this work.

\section{REFERENCES}

[1] Matthew W. Klope, Robert C. Beason, Timothy J. Nohara, Micheal J. Begier, 'Role of near-miss bird strikes in assessing hazards', Human Wildlife Conflicts, Vol. 3(2): pp. 208-216, 2009.

[2] Nohara T. J., Weber P., Unkrainec A. et al., 'An Overview of Avian Radar Developments - Past, Present and Future', Kingston: Bird Strike Conference, pp.10-13, 2007.

[3] Chen, W.S.[Wei-Shi], Ning, H.S.[Huan-Sheng],Li,J.[Jing], 'Detecting and tracking of small moving target in avian radar images', IASP10,pp. 473-476, 2010.

[4] Xiao benlina, li fangfang b, mao xingliangc, jin huazhongb, 'Study on independent component analysis' application in classification and change detection of multispectral images', The international archives of the photogrammetry, remote sensing and spatial information sciences. Vol. XXXVII. Part b7, 2008.

[5] Hyavrinen A., 'Fast and Robust Fixed-Point Algorithms for Independent Component Analysis', IEEE Transactions on Neural Networks, Vol. 10(3): pp. 626-634,1999.

[6] Hyävrinen A., E. Oja., 'Independent component analysis: algorithms and applications', Neural Networks, Vol. 13(45): pp.411-430, 2000

[7] Tomas Zeman, 'Newton's method for Fast ICA algorithm', 2000 .

[8] Liu N. N. and Li J. W., 'A New Detection Algorithm Based on Cfar for Radar Image with Homogeneous BackGround', Progress in Electromagnetics Research C, Vol. 15, pp.13-22, 2010.

[9] John Weatherwax 'An Improved Method for Detection of Stationary Targets in High Clutter SAR Images', IEEE Transactions on Aerospace And Electronic Systems, Vol. 1, No. 13, 2005.

[10] Chen J., Leung H., Lo T., et al. A Modified Probabilistic Data Association Filter in Real Clutter Environment. IEEE Transactions on Aerospace and Electronic Systems, vol. 32(1): 300-314, 1996 